\title{
Interactive comment on "Assessment of natural and anthropogenic aerosol air pollution in the Middle East using MERRA-2, CAMS data assimilation products, and high-resolution WRF-Chem model simulations" by \\ Alexander Ukhov et al.
}

Anonymous Referee \#2

Received and published: 21 March 2020

\section{General comments}

The manuscript by Ukhov et. al., presents a detailed comparison of WRF-Chem, 
optical depth, $\mathrm{PM}_{10}, \mathrm{PM}_{2.5}$ and their spatial and temporal patterns. In addition to the comparison, the composition of aerosol among dust, sulfate, sea salt and other constituents have been discussed. The impact on aerosol air pollution has also been investigated. This study could have been completed by also including some comparison for vertical profiles of aerosol extinction or various components of aerosol (e.g. dust, sulfate), with the measurements (if available) or at least among the model and assimilated products. I have a few major and several minor concerns with the manuscript, which upon being addressed, I recommend publication in ACP.

\section{Major issues:}

1. The manuscript primarily focusses on the various aerosol product and WRFChem code has been modified to calculate these parameters. In section 4.2, authors mention that the code modification will be published in a forthcoming publication. Since the data produced for use in this publication is simulated with modified model code, yet not peer-reviewed, I can only recommend publication after the technical publication.

2. For sections 2.2, 5.2.2 and 5.2.3 authors use the MODIS combined deep blue (DB) and dark target (DT) product. It a level 3 gridded product at a much coarse spatial resolution of $1^{\circ} \times 1^{\circ}$. DB has poorer performance over water, while DT has limitation over land. In my opinion, authors should use separate DB and DT, level 2 gridded products, which are available at much finer $(10 \mathrm{~km} \times 10 \mathrm{~km})$ resolution (comparable to WRF-Chem and MAIAC). Moreover, level 2 product also allows the possibility of applying a quality assurance criterion, which has shown improvement in the comparison previously (for e.g. Liu, N., et al. (2019)).

3. For comparison with MODIS data products, the model data should be sampled around satellite overpass or at most averaged $\pm 1 \mathrm{~h}$ around satellite overpass. 
Further, in order to avoid sampling bias, only those days should be considered for calculating seasonal means when both measurements (AERONET/Satellite) and model (or assimilation) data are available.

4. The introduction is very long in general and can be curtailed by only keeping the content most important to the study. Some (not all) suggestions: - Lines 55-60MISR, AVHRR and CALIOPE are not relevant to this study. Some restructuring is also needed. For example, the description of the work to be presented in the text fits better towards the end of the introduction. In line 75, authors mention about evaluation to be presented in the subsequent section but this is followed by further literature review. Line 93 again starts with the highlight of work to be presented in this work.

5. The conclusion needs to be curtailed. Redundancy in the conclusion can be reduced. Some examples (not all): Lines 558-559 and lines 571-543; Lines 560561 and 574-575. Numbers should be provided in conclusion rather than only qualitatively stating "overestimate" / "contribute" etc.

\section{Detailed Comments:}

1. Abstract: Please use abbreviations only after providing their full form at the first use (e.g. ME). Abstract does not do justice to the manuscript. Some more key finding should be added.

2. Line 6: WRF-CHEM code was modified but this is not described in detail in this manuscript. Authors wish to publish it as separate manuscript and hence this does not fit to abstract.

3. Line 15: rich - reach 
4. Lines 15-16: Contribution of both organic matter and black carbon are negligible. Is is important to mention this comparison?

5. Line 35: Essential - Important/crucial

6. Line 46: $\mathrm{PM}_{10}$ and $\mathrm{PM}_{2.5}$ are defined with respect to "aerodynamic diameter".

7. Lines 61-63: Is it justified to compare the 21 days' mean with air quality regulation standards for 1 year. Please note that some of the measured mean $\mathrm{PM}_{10}$ concentrations are smaller than 24 hours' limit.

8. Lines 79-85: What are the conclusions of these comparisons?

9. Lines 89-92: Given that mineral dust contributes $75-95 \%$ of the PM, how much discrepancy is caused by outdated emission inventories in MERRA-2 and CAMSOA?

10. Line 113: What are CIMEL and PREDE?

11. Lines 118-120: Authors should also provide a statistical comparison for the case when only cloud screened and quality-assured data are used in the results and discussion.

12. Line 119, 122: Angstrom - Ångström

13. Line 139: MAIAC also provides AOD at $470 \mathrm{~nm}$.

14. Section 2.2: Please mention the Quality assurance filter criteria if applied!

15. Line 153 ? quarterly refers to what?

16. Line 164 (DMS)

17. Line 173: This line is not clear to me. 
18. Line 179 and later in the text: dustbins - dust-bins.

19. Line 2019: OH is hydroxyl radical and not "Hydroxide radical".

20. Line 219: I had difficulties understating the treatment of PM, BC and OC emissions. Black carbon, organic carbon and dust, these are already included in PM. So if the emission of both PM and its constituents are specified separately, this would end up in doubling of certain constituents of PM.

21. Equation 3: Use of $S$ in both LHS and RHS are confusing. I would suggest using Smod or S' or something different.

22. Lines 250-253: How is the value of $C=0.5$ achieved? The tuning of $C$ with respect to measured AOD should be discussed in more detail.

23. Section 4.2: How are the diagnostic output of PM are different from those calculated in section 5.3 ?

24. Section 5.1 Lines 271-275 fit better for methods/domain description.

25. Figure 2: What is the physical significance of the topographic source function? Do the high values represent higher dust emission potential?

26. Line 283: Missing “)”.

27. Lines 305-309: Higher R and lower RMSD for $\mathrm{V}$ are not specific only for summers.

28. Table 3: How are the statistics for Autumn and Spring

29. Table 3,4 and 5: Slope/Bias should also be provided in addition to the $\mathrm{R}$ and RMSD. These quantities provide an idea about overestimation/underestimation/trend. 
30. Line 315: Aerosol content is also characterized by other quantities apart from AOD.

31. Lines 327-330: It would be nice to see the underestimation/overestimation with default $\mathbf{s}_{p}$ fraction and its magnitude as a figure (at least in appendix).

32. Line 345: This line should only be kept if the evaluation of updated CAMS-OA is shown in the manuscript.

33. Figure 6: Please mention that panel A corresponds to 2015 and B correspond to 2016.

34. Line 366: At a given location, up to 4 measurements are possible on several days due to overlap of two orbits each for TERRA and AQUA.

35. Section 5.2: I was surprised to see that MAIAC underestimates AOD with respect to AERONET. The evaluation of MAIAC by Lyapustin et. al., shows overestimation at all the three AERONET sites shows in this study. Authors should address, why even for a similar dataset, an underestimation is observed in this study by MAIAC. Authors could also refer to the finding of Liu et. al., 2019, where they have found that applying a QA filter significantly reduces the Deep blue (over land) AOD from MODIS over China. There are other evaluation studies (e.g. Liu et al., 2019, Mhawish et. al., 2019), which have found MAIAC to be more accurate than Deep blue and Dark Target. Authors should address, why for their domain this is not the case.

36. Figure 7 , I wonder how there are NAN values at around $40{ }^{\circ} \mathrm{N} 40^{\circ} \mathrm{E}$ in MODIS DB\&DT products in the annual mean but there are no NAN values in MAIAC annual mean. If the seasonal NAN values are removed by annual mean, this should hold valid for both the MODIS data products. I would recommend the authors to recheck the calculation of spatial means. Please also indicate the 
location of three AERONET site in Figure 7. This would help the reader to follow the discussion.

37. Section 5.3: Please provide references from where the formulas for calculation of $\mathrm{PM}_{2.5}$ and $\mathrm{PM}_{10}$ are adapted. What is the rationale behind the choice of the coefficients used in equation 4 and 5 ?

Interactive

comment

38. Sections 5.3, 5.3.3, 5.3.4 and 5.4: Air Quality and Air pollution are very broad terms which also include trace gases in addition to the aerosol. Hence, the subtitles of these sections should be made more specific.

39. Line 443: How does the calculated concentration of $298 \mu \mathrm{g} / \mathrm{m}^{3}$ compare against the measurements?

40. Figure 8 and 9: Please provide the uncertainty marks in the histogram which represent the variability over the mean.

41. Lines 496-503 Authors evaluate the $\mathrm{PM}_{2.5} / \mathrm{PM}_{10}$ ratio to evaluate the dominance of coarse/fine particles. A more quantitative evaluation would be $\mathrm{PM}_{10}-\mathrm{PM}_{2.5}$, which provides a more exclusive number for larger particles.

42. What are the major non-sulfate constituents in total $\mathrm{PM}_{2.5}$ non-dust aerosol?

43. Lines 587-588: In addition to the AOD retrieval uncertainty, there are several other differences e.g. Spatial resolution, Quality assurance filter which contribute the observed difference.

44. Line 600: Please use the same convention for the naming of seasons. "Fall" season is nowhere discussed in the text and appears for the first time in the conclusion.

45. Line 609: Air quality should be replaced with PM air quality. 


\section{References:}

1. Liu, N., et al. (2019), Evaluation and comparison of multiangle implementation of the atmospheric correction algorithm, Dark Target, and Deep Blue aerosol products over China, Atmos. Chem. Phys., 19(12), 8243-8268, doi:10.5194/acp19-8243-2019.

Interactive

comment

2. Mhawish, A., et al. (2019), Comparison and evaluation of MODIS Multiangle Implementation of Atmospheric Correction (MAIAC) aerosol product over South Asia, Remote Sensing of Environment, 224, 12-28, doi:10.1016/j.rse.2019.01.033.

3. Lyapustin, A., et al. (2018), MODIS Collection 6 MAIAC algorithm, Atmos. Meas. Tech., 11(10), 5741-5765, doi:10.5194/amt-11-5741-2018. 